\title{
Old muscle in young body: an aphorism describing the Chronic Fatigue Syndrome
}

Tiziana Pietrangelo $(1,2)$, Stefania Fulle $(1,2)$, Francesco Coscia $(3,4)$, Paola Virginia Gigliotti $(3,4)$, Giorgio Fanò-Illic $(1,2,5,6)$

(1) Department of Neuroscience Imaging and Clinical Sciences, G. d'Annunzio University of Chieti-Pescara, Chieti, Italy (2) IIM-Interuniversity Institute of Myology, Italy; (3) Sport Medicine Service of Autonome Provinz Bozen, San Candido-Innichen, Italy; (4) Laboratory of Sport Physiology, San Candido-Innichen, Italy; (5) A\&C M-C Foundation for Translational Myology, Padova, Italy; (6) Free University of Alcatraz, Santa Cristina di Gubbio, Italy.

This article is distributed under the terms of the Creative Commons Attribution Noncommercial License (CC BY-NC 4.0) which permits any noncommercial use, distribution, and reproduction in any medium, provided the original author(s) and source are credited.

\begin{abstract}
The chronic fatigue syndrome (CFS) otherwise known as myalgic encephalomyelitis (ME), is a debilitating syndrome whose identification is very complex due to lack of precise diagnostic criteria. This pathology begins with limitations in duration and intensity of exercise and rapid onset of pain during physical activity. Its etiology is unknown, and symptoms are not limited to the muscles. Epidemiology is rather difficult to delimit, even if it affects mainly young (20-40 years), female subjects. The results of muscular research show some peculiarities that can justify what has been observed in vivo. In particular, 1. presence of oxidative damage of lipid component of biological membranes and DNA not compensated by the increase of the scavenger activity; 2. Excitation-Contraction (E-C) alteration with modification of $\mathrm{Ca}^{2+}$ transport; 3. passage from slow to fast fiber phenotype; 4. inability to increase glucose uptake; 5. presence of mitochondrial dysfunction; and 6. genes expressed differentially (particularly those involved in energy production). The skeletal muscles of CFS / ME patients show a significant alteration of the oxidative balance due to mitochondrial alteration and of the fiber phenotype composition as shown in sarcopenic muscles of the elderly. Vice versa, the muscle catabolism does not appear to be involved in the onset of this syndrome. The data support the hypothesis that patients with CFS are subjected to some of the problems typical for muscle aging, which is probably related to disorders of muscle protein synthesis and biogenesis of mitochondria. Patients with CFS can benefit from an appropriate training program because no evidence suggests that physical exercise worsens symptoms. Type, intensity and duration of any physical activity that activates muscle contraction (including Electrical Stimulation) require further investigation even if it is known that non-exhaustive physical activity decreases painful symptomatology.
\end{abstract}

Key Words: Chronic Fatigue Syndrome, Myalgic Encephalomyelitis, Systemic Exertion Intolerance Disease, skeletal muscle, painful symptomatology, physical exercise

Eur J Transl Myol 28 (3): 239-247, 2018

Chronic Fatigue Syndrome (CFS) otherwise known as Myalgic Encephalomyelitis (ME) is a clinically defined condition characterized by persistent, severe, disabling fatigue lasting more than 6 months that is not reversed by sleep or rest. ${ }^{1}$ It is characterized by the onset of muscular fatigue and pain even after exercise (especially aerobic) of limited intensity and duration. Its etiology is unknown, and symptoms are not limited to the muscles only. The incidence of the disease is rather difficult to define, even though it seems to affect predominantly young females (20-40 years). The duration of illness typically exceeds 10 years and such pathology is quite common. In the UK, the annual incidence of recorded cases is currently 14.8 per 100,000 people. Annual incidence rates for CFS / ME diagnoses decreased from 17.5 in 2001 to 12.6 in 2013 with an annual percent change of $-2.8 \%$. $^{2}$ The degree of development of the disease is variable, and there is no common progression for all those affected. It is of unknown etiology, and it is associated with many 
Diagnosis requires that the patient have the following three symptoms:

1. A substantial reduction or impairment in the ability to engage in pre-illness levels of occupational, educational, social, or personal activities, which persists for more than 6 mo. and is accompanied by fatigue, which is often profound, is of new or definite onset (not lifelong), is not the result of ongoing excessive exertion, and is not substantially alleviated by rest

2. Post exertional malaise

3. Unrefreshing sleep

At least one of the two following manifestations is also required:

1. Cognitive impairment

2. Orthostatic intolerance

Frequency and severity of symptoms should be assessed. The diagnosis should be questioned if patients do not have these symptoms at least half of the time with moderate, substantial, or severe intensity

symptoms of non-muscular origin. Because of this unclear situation (Table 1.), the Institute of Medicine, National Academy, Washington, DC, USA in 2015 proposed to change the name of the disease as Systemic Exertion Intolerance Disease (SEID). ${ }^{3}$ There is no specific test yet to confirm a diagnosis of chronic fatigue syndrome. A variety of medical tests may be needed to rule out other health problems that have similar symptoms. In addition to premature muscle fatigue, signs and symptoms may include memory loss, unexplained muscle or joint pain, not-restorative sleep, extreme exhaustion that lasts more than 24 hours after exercise. Moreover, this chronic disability has farreaching consequences and represents a significant public health concern and an economic burden to the society. Some people may be born with predisposition for the disorder, and it can be triggered by a combination of factors such as:

1. Viral infections. It has been proposed that some viruses, such as Epstein-Barr virus, human herpesvirus 6 , could trigger the disorder. ${ }^{4}$

2. Deficiency of the immune system. Many CFS patients show atypical circulating lymphocytes and increased plasma level of C-reactive protein and other markers of immune impairment. ${ }^{5}$

3. Hormonal imbalances. Patients with a diagnosis of CFS / ME very often present abnormal levels of hormones produced in the hypothalamus, hypophysis or adrenal glands. ${ }^{5}$ In fact, there is a hypothalamic-pituitary-adrenal (HPA) axis hypoactivity state as indicated by the low basal glucocorticoid levels in these patients. As a consequence of this, the possibility of bidirectional communication between the HPA axis and the immuno-inflammatory system exists. Unfortunately, no cause-effect relationship between the two mechanisms has been determined. ${ }^{6}$

4. Psychological impairment. Cognitive-behavioral models of CFS suggest that personality factors may contribute to the syndrome development. ${ }^{7}$
The complex clinical picture and the profound disagreement on the potential pathogenic mechanism make ME / CFS a controversial pathology especially in the absence of specific biomarkers of the disease that would be of great help in its diagnosis and subsequent management. The biomarker by definition should include both sensitive and specific markers for the diagnosis of ME / CFS and those able to classify the subtypes of the disease to be also used as a prognostic indicator. An attempt to solve this problem is currently underway thanks to the EUROMENE program, which is a network of researchers and physicians of the European COST program within Horizon 2020 (http://www.cost.eu/COST_Actions/ca/AC15111). The objectives of EUROMENE are to promote strategies for collaboration and harmonization of diagnosis and research and to compile a database of clinical and scientific data consistent with ME / CFS presence. ${ }^{8}$ Also for these reasons, the CFS treatment is very difficult to outline. Trials of Rintatolimod (a drug able to protect and stimulate the nonspecific immune system), counseling therapies, and graded exercise therapy exert benefit for some patients (meeting case definitions for CFS), whereas evidence for other treatments and harms is insufficient. ${ }^{9}$. More definitive studies comparing participants that meet meeting different pathological definitions, including CFS, are needed to fill research gaps. ${ }^{10-13}$. Until specific molecular and/or biochemical characteristics are determined, the efforts to develop effective treatments for CFS will continue to be not conclusive.On the other hand, the clinical benefits of physiotherapy approaches developed for other similar clinical cases of early aging and aging per se (whether traumatic, metabolic, genetic or equally unknown), justify the option to apply them to CFS. ${ }^{14-16}$ In summary, to date, the number of studies performed to define the clinical aspects of the disease is still not adequate to clearly indicate onset and prognosis. ${ }^{17}$ Probably, its persistence may involve complex interactions of immune, autonomic and neuroendocrine regulation but 


\section{Chronic Fatigue Syndrome / Myalgic Encephalomyelitis}

Eur J Transl Myol 28 (3): 239-247, 2018

these studies remain poorly understood. ${ }^{18} \mathrm{~A}$ substantial part, even if minority, of the scientific research studied the biochemical-functional alterations that occur in the muscles of patients affected by CFS. The results highlighted some peculiarities that can justify what "in vivo" was observed. In particular:

- the presence of significant oxidative damage to lipids and DNA molecules with consequent alteration of cell membrane capacity not offset by the increase of the activity of endogenous antioxidant systems. ${ }^{19}$

- a negative influence on E-C cycle due to an alteration of opening RyR-channels status and a deregulation of $\mathrm{Ca}^{2+}$-ATPase pump. ${ }^{20}$

- a shift from slow to the fast twitch fiber phenotype with an increase in the energetically expensive fibers. $^{21}$

- the presence of mitochondrial dysfunction highlighted by lowered ATP production, impaired oxidative phosphorylation, and presence of mitochondrial damage. ${ }^{22}$

- the impairment of AMPK activation in primary CFS / ME skeletal muscle cell cultures derived from patients in response to electrical stimulation. This abnormality in AMPK activation also resulted in failure to increase glucose uptake into the cell in response to EPS. ${ }^{23}$

Also interesting are data of transcriptome analysis carried out to identify genes that were differentially expressed in muscles derived from CFS / ME patients. In the last decade, some research has highlighted that genes that play key roles in mitochondrial function (in particular that involved in energy production), oxidative balance, fiber phenotype were negatively altered with respect to controls in such way measurable in sarcopenic muscles. ${ }^{24}$ Vice versa, muscle tropism does not seem to be involved in the onset of fatigue syndrome because some of the genes involved in the processes leading to atrophy (FOXO, ubiquitin) are slightly downregulated in the samples coming from the muscles of CFS / ME patients. ${ }^{21}$. However, the associations found can all be assigned to 17 transcripts related to the basic cellular processes involved in signal transduction, ion transport and immune system function. ${ }^{25}$

Taken together, these results support the hypothesis that in CFS / ME patients the skeletal muscle undergoes some aspects of an involution typical of aging (sarcopenia) and this fact could determine the subsequent disease development. ${ }^{26,27}$ This hypothesis will also be discussed using data from the most recent literature on the specific metabolic-functional muscle markers of CFS comparing them with those derived from sarcopenic muscles. ${ }^{28-30}$

\section{Metabolic and functional CFS / ME markers}

Aiming to investigate the kinetics of denervationinduced One of the early and common symptoms of all the patients with CFS / ME diagnosis is the presence of a generalized feeling of abnormal fatigue, which is evident even after a relatively short and/or low-intensity physical activity. Although not all authors are of the same opinion (agree on this), the origin of fatigue does not seem to be central but rather peripheral. ${ }^{31-33}$ Generally, describing their fatigued state, almost all the subjects analyzed indicated a precise sensation of "lack of muscular energy". This feeling seems to be confirmed by recent studies of Magnetic Resonance Spectroscopy that indicate significant bioenergetic abnormalities in the muscles of CFS patients. ${ }^{34}$ Thus contradicting previous observations that did not show, in the muscles of similar patients, metabolic alterations subsequent to physical exercise. ${ }^{35}$ The decrease in ATP production also demonstrated the presence of muscle damage due to the impaired oxidative phosphorylation and the consolidated mitochondrial damage. More recently, post-exercise alteration of AMP-activated protein kinase, involved in the stimulation of skeletal muscle fatty acid oxidation and glucose uptake has also been demonstrated. ${ }^{36}$ Skeletal muscle is energetically very expensive, and utilizes glucose and fatty acids using nicotinamide adenine oxidized dinucleotide $\left(\mathrm{NAD}^{+}\right)$and its reduced form, NADH, for electron transfer. $\mathrm{NAD}^{+}$levels influence many different cellular mechanisms such as mitochondrial biogenesis, gene transcription and extracellular matrix organization. ${ }^{29}$ Considering that the overwhelming majority of studies indicate that low $\mathrm{NAD}^{+}$levels are detrimental to skeletal muscle integrity while higher $\mathrm{NAD}^{+}$levels increase muscle functional capacity, ${ }^{37}$ it is possible to speculate that $\mathrm{NAD}^{+}$(or rather its cellular expression) is one of the main culprit involved in aging and in many diseases (included CFS / ME) of skeletal muscles. Considerable evidence indicates that mitochondrial dysfunction with resulting oxidative damage contribute to the sarcopenic phenotype that occurs with aging. ${ }^{29}$ As a consequence, many structural and functional changes occur with advancing age in skeletal muscle, including a reduction in the number and cross-sectional area (CSA) of muscle fibers. ${ }^{38}$ A similar scenario can also be observed in samples derived from CFS / ME subjects in which experiments of functional analysis of the fibers coming from the quadriceps muscle showed some morphofunctional alterations. In a case-control study, the phenotype of the same biopsy samples was analyzed to determine: i) fiber-type proportion using myosin isoforms as fiber-type molecular marker and gel electrophoresis as a tool to separate and quantify myosin isoforms, and ii) contractile properties of manually dissected, chemically made permeable and calciumactivated single muscle fibers. The results derived from these experimental procedures showed that the fibertype proportion was significantly altered in CSF samples (a significant shift from the slow- to the fasttwitch phenotype). Vice versa, cross-sectional area, force, maximum shortening velocity and calcium 


\section{Chronic Fatigue Syndrome / Myalgic Encephalomyelitis}

Eur J Transl Myol 28 (3): 239-247, 2018

sensitivity were not significantly changed in single muscle fibers from CSF samples. Thus, the contractile properties of muscle fibers were preserved, but their proportion was changed, with an increase in the more fatigue-prone, energetically expensive fast fiber type. ${ }^{21}$ Partially contradictory data, at least with regards the type of fiber prevalent in the quadriceps of CFS muscles, had been obtained from Lane et al. ${ }^{35}$ using a semi-automated image analysis system and Electron Microscopy techniques. However, in the same paper, the authors say that patients with increased lactate responses to exercise, did have significantly fewer type 1 muscle fibers, but there was no evidence that this group was less active than the patients with normal lactate responses.

\section{Oxidative Stress in CFS / ME muscles}

To establish which are elements not common but peculiar for CFS with respect to other similar pathologies, we will start by analyzing the specific muscle components linked to the excitation-contraction cycle, since the apparatus generating force and the neuromuscular junction did not seem to undergo changes directly related to the presence of the disease. In particular, the consequence of mitochondrial dysfunction could be an increased production of Reactive Oxygen Species (ROS). ROS are generated by the addition of a single electron to the oxygen molecule and are formed in all tissues including muscle fibers in the mitochondrial respiratory chain. Such reactive elements are usually quite harmful and result in oxidative stress that can damage many cellular components such as DNA, proteins, lipids. Considerable evidence has shown that mitochondrial oxidative damage can alter mitochondrial integrity and function in aging skeletal muscle. This includes a reduction in mitochondrial abundance and oxidative phosphorylation, accumulation of mutated mitochondrial DNA associated with impaired autophagy, and increased mitochondrial-mediated apoptosis, which all could contribute to sarcopenia. Although cumulative oxidative damage has been suggested to induce age-associated decline in mitochondrial function, the effects of mitochondrial dysfunction and mtROS in age-related muscle atrophy remains a controversial topic. ${ }^{38}$ Elevated ROS levels have been associated, in CFS / ME muscles, with alterations in mitochondrial function with a consequent reduction in the rate of ATP synthesis and impairment of cellular bioenergetics and, as consequence of this to muscle fatigue. The molecular cause of this event is probably linked to the oxidation of at least two substrates: membrane lipids and DNA also due to a certain inefficiency of the scavenger and/or repair in mitochondria. ${ }^{39}$ In addition, muscle mitochondria are one of the main sources of ROS generation in cells and are therefore highly sensitive to oxidative damage. It has been previously found that age-dependent increase in oxidative damage in the muscle of healthy subjects may reflect either increased production of free radicals or insufficient activity of scavenger/repair systems. ${ }^{40}$ In particular, the authors examined markers of oxidative damage to DNA, lipids, and proteins in 66 muscle biopsy specimens from humans aged 25 to 93 years. There were age-dependent increases in a marker of oxidative damage to DNA and lipid peroxidation, and to a lesser extent in protein carbonyl groups, a marker of protein oxidation. These results provide evidence for a role of oxidative damage in muscle aging which may contribute to age-dependent losses of muscle strength and stamina. The same group also demonstrated specific oxidative alterations in the vastus lateralis muscle of CFS patients, both as an increased value of oxidative damage markers (8OH-dG, MDA) and membrane fluidity, and an imbalance in the oxidant-antioxidant system. ${ }^{19}$ These findings are in agreement with the hypothesis that impairment of mitochondrial activity underlies an increase in the production of ROS that leads to muscle fatigue, similar to normal ageing. ${ }^{41}$ In the muscles of CFS / ME patients, many of the reported alterations may be the consequence of oxidative damage. The data published by Fulle et al. ${ }^{20}$ seem to support this hypothesis and seem to indicate that the presence of oxidative stress alters the fluidity of the network of muscle membranes. It is possible that the excitation-contraction coupling, the fundamental mechanism of muscle contractions, is altered in this pathology. In particular, the sarcolemma conduction system and the calcium ion release/uptake mechanism are negatively affected in CFS / ME preparations. In fact, the activities of the sarcolemma $\mathrm{Na} / \mathrm{K}$ pumps and that of SERCA of the sarcoplasmic reticulum undergo deregulation in the muscles coming from CFS / ME patients compared to controls of the same age. Also the RYR channels are altered with consequent adverse effects on the transport of $\mathrm{Ca}^{2+}$ and possible accumulation of the ion in the sarcoplasm. This would lead to a state of contracture similar to what occurs during muscle fatigue. ${ }^{20}$ As occurs in the muscles of patients with CFS / ME, even in the vastus lateralis of elderly subjects, an alteration of the mechanism that controls the release of calcium from internal reserves has been proposed to explain the decline in muscle performance related to age. In a study by Boncompagni et al. $^{42}$ the frequency, cellular localization and ultrastructure of calcium release units were studied in male and female human muscle biopsies aged between 28 and 83 years. The results show significant alterations in the morphology and cell disposition of calcium release units and a significant decrease in their frequency as a function of the age of the subjects examined. This means that in humans the coupling apparatus undergoes a partial disarrangement and a spatial reorganization that could interfere with a valid release of $\mathrm{Ca}^{2+}$ ions to the contractile proteins. ${ }^{42}$ 


\section{Chronic Fatigue Syndrome / Myalgic Encephalomyelitis}

Eur J Transl Myol 28 (3): 239-247, 2018

\section{Gene modifications in CFS / ME muscles}

Recent studies carried out to characterize the differences in gene transcription in the muscles of patients diagnosed with Gulf War syndrome vs. CFS / MS patients have shown significant different profiles (such as to suggest a real difference at least from the genetic point of view), not only between the two pathologies but also between these and healthy controls. Significant is that about 50 genes responded, at peak maximum effort with respect to rest, in Gulf War samples, but only 1 was expressed in CFS / ME. Furthermore, most of the 466 genes expressed with a difference of 2 times (FDR $<0.05$ ) in CFS / ME compared to control subjects, were those directly or indirectly connected with the mechanisms of signal transduction and regulation of cell metabolism. ${ }^{43}$ Broderick et al. $^{25}$ analyzed correlation patterns in 117 clinical variables measured in 111 female subjects and used them to isolate the CFS / ME gene co-expression patterns. The associations found can all be assigned to 17 transcripts related to the basic cellular processes involved in signal transduction, ion transport and immune system function. Apparently, the most influential single gene found among those tested was identified with sestrin 1, which supports the involvement of oxidative stress in CFS / ME. Kerr et al. ${ }^{44}$ in the effort to search for "marker genes" with predictive power against CFS / ME, isolated a set of 44 genes to discriminate between CFS / ME patients and healthy control individuals. This classification was able to correctly discriminate between CFS / ME and healthy control samples in $95 \%$ of the training samples but was criticized for the difficulty of reproducing the results. More recently, Broderick et al. ${ }^{45}$ also demonstrated that differences in gene expression are related to 90 pathways, most of which are linked to immune metabolism. Examining these pathways as part of an integrated biological system would result in significant differences between CFS / ME patients and healthy control subjects. Indeed, these significant differences in regulatory interactions could not be observed using conventional analytical methods focused on the expression of individual markers. Utah researchers with access to computerized genealogical resource linking multiple generations of genealogy data with medical diagnosis data reported significant evidence for a heritable contribution to predisposition to CFS / ME. ${ }^{46}$ In addition, in order to establish which mechanisms and/or pathways could be involved in the development of typical CFS muscle symptoms, a global transcriptome analysis was used by Pietrangelo et al. ${ }^{24}$ to identify the genes that were expressed differentially in the vastus lateralis muscle of patients with positive diagnosis of CFS / ME. The analysis of the data obtained seems to clearly indicate that the expression of genes that play key roles in mitochondrial function and in oxidative equilibrium, including those that code for superoxide dismutase 2, appear modified, as well as seems to be altered in their expression also other genes involved in energy production, muscular trophism and the determination of fiber phenotype.The analysis of the correlation between gene expression of muscle mass and muscle functional changes with respect to age has identified genetic markers of sarcopenia distinct from those of general aging. In particular, some of the mitochondrial energy metabolism genes (tricarboxylic acid cycle and oxidative phosphorylation) are downregulated in the sarcopenic muscle. Furthermore, the gene expressions of the neuromuscular junction patency, those of protein catabolism and inflammation are also modified. ${ }^{47}$ These data suggest a strong similarity between what happens in the aging muscle with that which occurs in younger CFS / ME patients (Figure 1).

\section{Palliative Therapies}

CFS / ME is undoubtedly an emerging and challenging medical condition, but it is not without hope. At the moment, present there is no cure: the treatment simply aims to alleviate the symptoms and reverse muscle atrophy related to impaired mobility. In general, CFS / ME patients who are diagnosed within the first 2 years after the appearance of the symptoms respond better to treatment than those diagnosed later. Treatments to relieve symptoms must be personalized for each patient as it occurs for the management of age-related sarcopenia. ${ }^{48-50}$ Most specialists agree that CFS / ME patients need treatment based on a multidisciplinary and integrative approach. Some researchers suggest that a healthy diet and the use of food supplements are essential components of any therapeutic approach mainly due to the numerous gastrointestinal problems occurring in CFS / ME patients that can lead to inflammation and malabsorption. For these reasons, the CFS / ME treatment is very difficult to outline. Trials of Rintatolimod (a drug able to protect and stimulate the nonspecific immune system), counseling therapies, and graded exercise therapy suggest benefit for some patients which meets the case definitions for CFS, whereas evidence for other treatments and harms is insufficient. $^{51}$ More definitive studies comparing participants meeting different pathological definitions, including CFS, are needed to fill research gaps. Until specific molecular and/or biochemical characteristics are determined, the efforts to develop effective treatments for CFS will continue to be not conclusive. Rituximab is a monoclonal antibody active against CD20, a B ${ }^{+}$cell receptor. Rituximab works by depleting $\mathrm{B}^{+}$cells, thus reducing inflammation. Sustained overall improvements were noted in $67 \%$ of CFS / ME patients as opposed to $13 \%$ of controls. ${ }^{52}$ The UK National Institute of Health and Clinical Excellence recommends cognitive behavioral therapy (CBT) and graded exercise therapy (GET). Although this recommendation was supported by systematic reviews, supporting evidence remains limited to the small studies. A recognition conducted by patient organizations in the UK have 


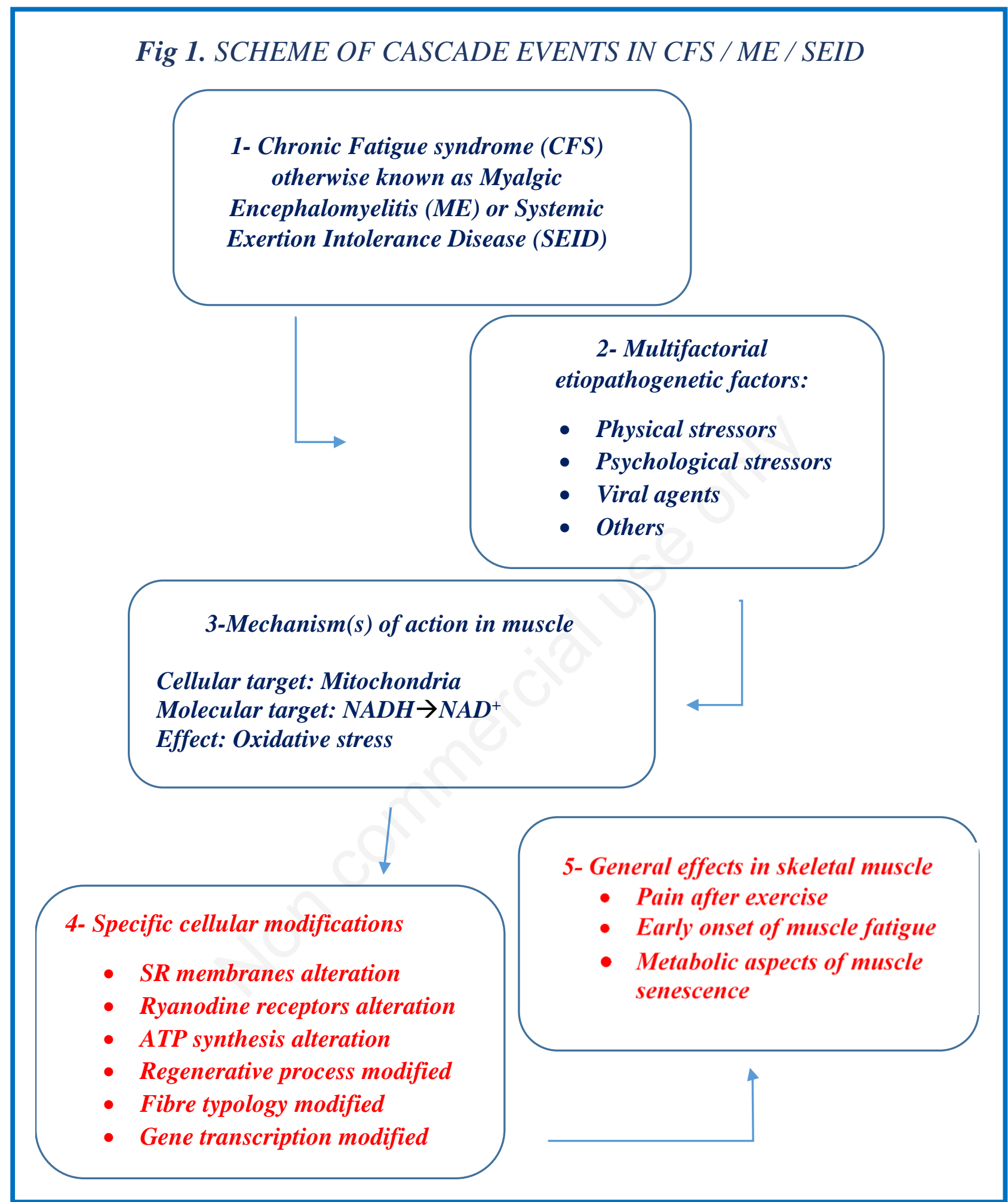

reported that CBT and GET are sometimes harmful and have recommended pacing and specialist health care. To verify, by means of a comparative analysis, the efficacy of these methods when applied alone or in combination, White et al designed a randomized evaluation study PACE to compare the effects of pacing, defined as adaptive stimulation therapy (APT), CBT and GET with the addition of specialist medical care (SMC) vs SMC alone. The results show that CBT and GET would be more effective than APT and SMC, but that APT would be more effective than SMC alone. ${ }^{53}$ Antioxidants (including $\alpha$-lipoic acid vitamin $\mathrm{E}$ or $\mathrm{C}$ ) are a group of vitamins, minerals, and enzymes that help protect cells from damage caused by oxidative stress and also 


\section{Chronic Fatigue Syndrome / Myalgic Encephalomyelitis}

Eur J Transl Myol 28 (3): 239-247, 2018

improve mitochondrial function. ${ }^{54} 38$ consecutively diagnosed CFS women were treated with a multivitamin and mineral supplement for 2 months with follow-up. There was a significant improvement in the activity levels of superoxide dismutase, significant decreases in fatigue, sleep problems, symptoms of autonomic dysfunction, frequency and intensity of headaches and subjective feeling of infection. The conclusion was that such treatment could be a safe and simple way to improve symptoms and quality of life in CFS / ME patients. ${ }^{55}$ Coenzyme Q10 and $\mathrm{NADH}$ are common antioxidant supplements that are used as dietary supplements for general maintenance of health. However, several studies have shown that their use induces in CFS / ME (where a mitochondrial dysfunction, which reduces the ATP production exists) as an immediate effect primary or secondary to symptom reduction in most CFS / ME patients. ${ }^{56}$ In summary, to date, the number of studies performed to define the clinical aspects of the disease is still not adequate to clearly indicate onset and prognosis. Probably, its persistence may involve complex interactions of immune, autonomic and neuroendocrine regulation but these studies remain poorly understood. ${ }^{57}$

\section{Conclusions}

The purpose of this review was to summarize some of the peculiarities of the muscles of patients with CFS / ME or SEID diagnose. As it could help researchers and clinicians to understand and treat more appropriately both symptomatology and prophylaxis of such obscure conditions that is without certain origin and demonstrated etiopathogenetic agent(s). From the most recent literature, and specifically from the data obtained in authors' laboratories, the image of an "old muscle in a young body" is confirmed by numerous experimental observations. This means that more than a "disease", CFS / ME is a (mal)functional state that can be treated to alleviate the symptoms and not to cure it.

\section{List of acronyms}

APT - adaptive stimulation therapy

CBT - cognitive behavioral therapy

CFS - Chronic Fatigue Syndrome

CSA - cross-sectional area

E-C - Excitation-Contraction

EPS - electrical pulse stimulation

FDR - false discovery rate

ET - graded exercise therapy

HPA - hypothalamic-pituitary-adrenal

ME - Myalgic Encephalomyelitis

ROS - Reactive Oxygen Species

SEID - Systemic Exertion Intolerance Disease

SMC - specialist medical care

\section{Author's contributions}

GFI, SF and TP drafted the article. All authors revised the paper critically and approved the final typescript.

\section{Acknowledgments}

We thanks all collaborators in the laboratories of SF, TP and GFI.

Funding: Publication costs of this review were covered by the A\&C M-C Foundation for Translational Myology, Padova, Italy

\section{Conflict of Interest}

All authors declare no conflicts of interests.

\section{Ethical Publication Statement}

We confirm that we have read the Journal's position on issues involved in ethical publication and affirm that this report is consistent with those guidelines.

\section{Corresponding Author}

Prof. Giorgio Fanò-Illic, Free University of Alcatraz, Santa Cristina di Gubbio (Italy).

Email: fanoillic@gmail.com

\section{E-mail of co-authors}

Tiziana Pietrangelo: tiziana.pietrangelo@unich.it Stefania Fulle: s.fulle@unich.it

Francesco Coscia: francesco.coscial@gmail.com Paola Gigliotti: pao.gigliotti@gmail.com

\section{References}

1. Norheim KB, Jonsson G, Omdal R. Biological mechanisms of chronic fatigue. Rheumatology (Oxford) 2011;50:1009-18.

2. Collin SM, Bakken IJ, Nazareth I, et al. Trends in the incidence of chronic fatigue syndrome and fibromyalgia in the UK, 2001-2013: a Clinical Practice Research Datalink study. J R Soc Med 2017;110:231-44.

3. Clayton EW. Beyond myalgic encephalomyelitis/ chronic fatigue syndrome: an IOM report on redefining an illness. JAMA 2015;313:1101-2.

4. Nicolson GL, Gan R, Haier J. Multiple coinfections (Mycoplasma, Chlamydia, human herpes virus-6) in blood of chronic fatigue syndrome patients: association with signs and symptoms. APMIS 2003;111:557-66.

5. Morris G, Anderson G, Maes M. HypothalamicPituitary-Adrenal Hypofunction in Myalgic Encephalomyelitis (ME)/Chronic Fatigue Syndrome (CFS) as a Consequence of Activated Immune-Inflammatory and Oxidative and Nitrosative Pathways. Mol Neurobiol 2017;54:6806-19.

6. Ben-Zvi A, Vernon SD, Broderick G. Model-based therapeutic correction of hypothalamic-pituitaryadrenal axis dysfunction. PLoS Comput Biol 2009;5:e1000273.

7. Brooks SK, Chalder T, Rimes KA. Chronic Fatigue Syndrome: Cognitive, Behavioural and Emotional Processing Vulnerability Factors. Behav Cogn Psychother 2017;45:156-169. 


\section{Chronic Fatigue Syndrome / Myalgic Encephalomyelitis}

Eur J Transl Myol 28 (3): 239-247, 2018

8. Scheibenbogen C, Freitag H, Blanco J,et al The European ME/CFS Biomarker Landscape project: an initiative of the European network EUROMENE. J Transl Med 2017;15:162.

9. Smith ME, Haney $\mathrm{E}$, McDonagh $\mathrm{M}$, et al. Treatment of Myalgic Encephalomyelitis/Chronic Fatigue Syndrome: A Systematic Review for a National Institutes of Health Pathways to Prevention Workshop. Ann Intern Me. 2015;162:841-50.

10. Sajer S. Mobility disorders and pain, interrelations that need new research concepts and advanced clinical commitments. Eur J Transl Myol 2017;27:7179.

11. Gislason MK, Ingvarsson $P$, Gargiulo $P$, Yngvason S, Guðmundsdóttir V, Knútsdóttir S, Helgason P. Finite Element Modelling of the Femur Bone of a Subject Suffering from Motor Neuron Lesion Subjected to Electrical Stimulation. Eur J Transl Myol 2015;24(3):2187. doi: 10.4081/ejtm.2014.2187. eCollection 2014 Sep 23.

12. Carraro U. Muscle and skin improve by homebased FES and full-body in-bed gym. Biol Eng Med 2018;3:4-4.

13. Edmunds KJ, Gíslason MK, Arnadottir ID, et al. Quantitative Computed Tomography and Image Analysis for Advanced Muscle Assessment. Eur J Transl Myo 2016; 26:6015.

14. Coletti D. Exercise against tumor- and chemotherapy-induced muscle wasting. Biol Eng Med 2018; 3:1-5. doi: 10.15761/BEM.1000S1004.

15. Masiero S, Musumeci A. Rehabilitation medicine for elderly patients, a further note. Biol Eng Med 2018;3:1-2. doi: 10.15761/BEM.1000S1006.

16. Carraro U. The Giovanni Salviati Memorial and Translational Myology Audio Visual Abstracts at the 2018 SpPMD. ARC Journal of Research in Sports Medicine 2018;2:25-31.

17. Rutherford G, Manning $P$, Newton JL. Understanding Muscle Dysfunction in Chronic Fatigue Syndrome. J Aging Res 2016;2016:2497348. doi: 10.1155/2016/2497348

18. Klimas NG, Broderick G, Fletcher MA. Biomarkers for chronic fatigue. Brain Behav Immun 2012;26:1202-10.

19. Fulle S, Mecocci P, Fanó G, et al. Specific oxidative alterations in vastus lateralis muscle of patients with the diagnosis of chronic fatigue syndrome. Free Radic Biol Med 2000;29:1252-9.

20. Fulle S, Belia S, Vecchiet J, et al. Modification of the functional capacity of sarcoplasmic reticulum membranes in patients suffering from chronic fatigue syndrome. Neuromuscul Disord 2003;13:479-84.

21. Pietrangelo T, Toniolo L, Paoli A, et al. Functional characterization of muscle fibres from patients with chronic fatigue syndrome: case-control study. Int J Immunopathol Pharmacol 2009;22:427-36.
22. Filler K, Lyon D, Bennett J, et al. Association of Mitochondrial Dysfunction and Fatigue: A Review of the Literature. BBA Clin 2014;1:12-23.

23. Brown AE, Dibnah $\mathrm{B}$, Fisher $\mathrm{E}$ et al. Pharmacological activation of AMPK and glucose uptake in cultured human skeletal muscle cells from patients with ME/CFS. Biosci Rep 2018;38

24. Pietrangelo $\mathrm{T}$, Mancinelli R, Toniolo L, et al. Transcription profile analysis of vastus lateralis muscle from patients with chronic fatigue syndrome. Int $\mathrm{J}$ Immunopathol Pharmacol 2009;22:795-807.

25. Broderick G, Craddock RC, Whistler $\mathrm{T}$, et al. Identifying illness parameters in fatiguing syndromes using classical projection methods. Pharmacogenomics 2006;7:407-19.

26. Musarò A, Fulle S, Fanò G. Oxidative stress and muscle homeostasis. Curr Opin Clin Nutr Metab Care 2010;13:236-42.

27. Pellegrino MA, Desaphy JF, Brocca L, et al. Redox homeostasis, oxidative stress and disuse muscle atrophy. J Physiol 2011;589(Pt 9):2147-60.

28. Mosole S, Carraro U, Kern H, et al. Use it or Lose It: Tonic Activity of Slow Motoneurons Promotes Their Survival and Preferentially Increases Slow Fiber-Type Groupings in Muscles of Old Lifelong Recreational Sportsmen. Eur J Transl Myol 2016;26:5972. eCollection 2016 Sep 15; 12.

29. Yasir S. Elhassan, Andrew A. Philp, Gareth G. Lavery Targeting NAD+ in Metabolic Disease: New Insights Into an Old Molecule J Endocr Soc 2017; 1: 816-35.

30. Kern H, Carraro U. Home-Based Functional Electrical Stimulation for LongTerm Denervated Human Muscle: History, Basics, Results and Perspectives of the Vienna Rehabilitation Strategy. Eur J Transl Myol 2014;24:3296.

31. Staud R. Peripheral and central mechanisms of fatigue in inflammatory and noninflammatory rheumatic diseases. Curr Rheumatol Rep 2012;14:539-48.

32. Toussaint LL, Whipple MO, Abboud LL, et al. A mind-body technique for symptoms related to fibromyalgia and chronic fatigue. Explore (NY) 2012;8:92-8.

33. Jones DE, Hollingsworth $\mathrm{KG}$, Taylor $\mathrm{R}$, et al. Abnormalities in $\mathrm{pH}$ handling by peripheral muscle and potential regulation by the autonomic nervous system in chronic fatigue syndrome. J Intern Med 2010;267:394-401.

34. Jones DE, Hollingsworth $\mathrm{KG}$, Jakovljevic et al. Loss of capacity to recover from acidosis on repeat exercise in chronic fatigue syndrome: a casecontrol study. Eur J Clin Invest 2012;42:186-94.

35. Lane RJ, Barrett MC, Woodrow D et al. Muscle fibre characteristics and lactate responses to exercise in chronic fatigue syndrome. J Neurol Neurosurg Psychiatry 1998;64:362-7. 


\section{Chronic Fatigue Syndrome / Myalgic Encephalomyelitis}

Eur J Transl Myol 28 (3): 239-247, 2018

36. Brown AE, Jones DE, Walker M, Newton JL. Abnormalities of AMPK activation and glucose uptake in cultured skeletal muscle cells from individuals with chronic fatigue syndrome. PLoS One 2015;10:e0122982.

37. Goody MF, Henry CA. A need for NAD+ in muscle development, homeostasis, and aging. Skelet Muscle 2018;8:9.

38. Sakellariou GK, Pearson T, Lightfoot AP et al. Long-term administration of the mitochondriatargeted antioxidant mitoquinone mesylate fails to attenuate age-related oxidative damage or rescue the loss of muscle mass and function associated with aging of skeletal muscle. FASEB J 2016;30:3771-85.

39. Crane JD, Abadi A, Hettinga BP et al. Elevated mitochondrial oxidative stress impairs metabolic adaptations to exercise in skeletal muscle. PLoS One 2013;8:e81879.

40. Fanò $\mathrm{G}$, Mecocci $\mathrm{P}$, Vecchiet $\mathrm{J}$, et al. Age and sex influence on oxidative damage and functional status in human skeletal muscle. J Muscle Res Cell Motil 2001;22:345-51.

41. Mecocci P, Fanó G, Fulle S, et al. Age-dependent increases in oxidative damage to DNA, lipids, and proteins in human skeletal muscle. Free Radic Biol Med 1999;26:303-8.

42. Boncompagni S, d'Amelio L, Fulle S, et al. Progressive disorganization of the excitationcontraction coupling apparatus in aging human skeletal muscle as revealed by electron microscopy: a possible role in the decline of muscle performance. J Gerontol A Biol Sci Med Sci 2006;61:995-1008.

43. Klimas NG, Broderick G, Fletcher MA. Biomarkers for chronic fatigue. Brain Behav Immun 2012;26:1202-10.

44. Kerr JR, Petty R, Burke B, et al. Gene expression subtypes in patients with chronic fatigue syndrome/myalgic encephalomyelitis. J Infect Dis 2008;197:1171-84.

45. Broderick G, NG Klimas, MA Fletcher, S Efroni. Presentation to the Research Advisory Committee on Gulf War Veterans Illnesses; June 27-28; Washington, D.C. 2011b. http://www.va.gov/ RAC-GWVI/Minutes_June_2011.asp.).

46. Albright F, Light K, Light A, et al. Evidence for a heritable predisposition to Chronic Fatigue Syndrome. BMC Neurol 2011;11:62.

47. Ibebunjo C, Chick JM, Kendall T, et al. Genomic and proteomic profiling reveals reduced mitochondrial function and disruption of the neuromuscular junction driving rat sarcopenia. Mol Cell Biol 2013;33:194-212.
48. Barber L, Scicchitano, Musaro A. Mechanisms of Muscle Aging and Sarcopenia and Effects of Electrical Stimulation in Seniors. Eur J Transl Myol 2015;25:231-6.

49. Ricci G, Simoncini C, Baldanzi S and Siciliano G. Opportunities and potential applications from healthcare technologies to assist motor activity in metabolic myopathies. Biol Eng Med 2018;3:1-4.

50. Hofer C, Loefler S, Kern H, et al. Two years of FES training improves muscle fibers of thigh muscles in long-term thoracic level-complete spinal cord injury. Biol Eng Med 2018;3:1-5.

51. Smith ME, Haney E, McDonagh $M$, et al. Treatment of Myalgic Encephalomyelitis/Chronic Fatigue Syndrome: A Systematic Review for a National Institutes of Health Pathways to Prevention Workshop. Ann Intern Med 2015;162:841-50.

52. Fluge $\varnothing$, Risa K, Lunde S, et al. B-Lymphocyte Depletion in Myalgic Encephalopathy/ Chronic Fatigue Syndrome. An Open-Label Phase II Study with Rituximab Maintenance Treatment. PLoS One 2015;10:e0129898.

53. White PD, Goldsmith KA, Johnson AL et al. Comparison of adaptive pacing therapy, cognitive behaviour therapy, graded exercise therapy, and specialist medical care for chronic fatigue syndrome (PACE): a randomized trial. Lancet 2011;377:823-36.

54. Nicolson GL. Mitochondrial Dysfunction and Chronic Disease: Treatment With Natural Supplements. Integr Med (Encinitas) 2014;13:3543.

55. Maric D, Brkic S, Tomic S, et al. Multivitamin mineral supplementation in patients with chronic fatigue syndrome. Med Sci Monit 2014;20:47-53.

56. Castro-Marrero J, Sáez-Francàs N, Segundo MJ, et al. Effect of coenzyme Q10 plus nicotinamide adenine dinucleotide supplementation on maximum heart rate after exercise testing in chronic fatigue syndrome - A randomized, controlled, double-blind trial. Clin Nutr 2016;35:826-34.

57. Castro-Marrero J, Sáez-Francàs N, Santillo D, Alegre J. Treatment and management of chronic fatigue syndrome/myalgic encephalomyelitis: all roads lead to Rome. $\mathrm{Br} \mathrm{J}$ Pharmacol 2017;174:345-69.

Submission: July 7, 2018

Revision received: August 30, 2018

Acceptance: August 30, 2018 